\title{
IMMUNOLOGICAL AND BIOCHEMICAL CHARACTERISATION OF 7ap, A SHORT PROTEIN TRANSLATED FROM AN ALTERNATIVE FRAME OF ORF7 OF PRRSV
}

\author{
Ferenc OLASZ ${ }^{1 *}$, Béla DÉNES ${ }^{2}$, Ádám BÁLINT $^{2}$, Tibor MAGYAR $^{1}$, Sándor BELÁK ${ }^{3}$ \\ and Zoltán ZÁDORI ${ }^{1}$
}

${ }^{1}$ Institute for Veterinary Medical Research, Centre for Agricultural Research, Hungarian Academy of Sciences, Budapest, Hungary; ${ }^{2}$ Veterinary Diagnostic Directorate, National Food Chain Safety Office, Budapest, Hungary; ${ }^{3}$ Department of Biomedical Sciences and Veterinary Public Health (BVF), Swedish University of Agricultural Sciences (SLU), Uppsala, Sweden

(Received 22 January 2016; accepted 4 May 2016)

Sequence analysis revealed a short alternative open reading frame (ORF) named ORF7a within the nucleocapsid gene of genetically divergent porcine reproductive and respiratory syndrome virus (PRRSV) genomes. Alignment of the corresponding protein sequences (named 7ap) revealed substantial heterogeneity among 7aps of different genotypes, though all of them are predicted to be positively charged. Green fluorescent protein and FLAG fusion constructs of ORF7a of the HU-14432/2011 PRRSV demonstrated that 7ap is expressed. 7ap of HU14432/2011 (Hu7ap) was synthesised chemically, and ELISA experiments revealed that Hu7ap binds strongly to mammalian IgGs. Protein-protein gel retardation assays and complement fixation inhibition suggest that 7 aps bind to the $\mathrm{CH} 2$ domain of the $\operatorname{IgG}(\mathrm{Fc})$ fragment. Cellular localisation and immunological characteristics of PRRSV 7ap may indicate multiple functions including nuclear and cytoplasmic over-tuning of normal cellular processes and immunosuppression.

Key words: PRRSV, IgG binding, positive charged short peptide, overlapping ORF, complement fixation inhibition

Porcine reproductive and respiratory syndrome virus (PRRSV) is a member of the Arteriviridae family of the Nidovirales order. Since its appearance in the late 1980s PRRSV has remained one of the most costly diseases of the swine industry. PRRSV has a high mutation rate, which led to the evolution of two major genotypes with several subtypes (Murtaugh et al., 2010). The nucleotide identity between the two serotypes is only 55-70\%. Type I PRRSV, also known as the European genotype, is prevalent in Europe (although it may also cause infections in Asia and North America), while type II, also known as the North American genotype, causes infections mainly in China and North America (Stadejek et

*Corresponding author; E-mail: olasz.ferenc@agrar.mta.hu 
al., 2006; Shi et al., 2010). The high genetic diversity of PRRSV hinders the development of protective measures against the virus (Mateu and Diaz, 2008). Despite extensive scientific efforts and the substantial financial investments by stakeholders, currently there is no effective vaccine against the virus on the market (Zuckermann et al., 2007; Charerntantanakul, 2012; Tóth et al., 2016).

Several studies suggest that PRRSV can modulate the immune response of the host. Neutralising antibodies (NA) against PRRSV are generated in low levels in the early phases of the infection. Presence of non-neutralising antibodies in the early stages of the infection could enhance viral replication in alveolar macrophages due to antibody-dependent enhancement. The virus can evade the immune response of the host using several other strategies including delaying and inhibiting interferon response of the host cells, interfering with antigen presentation, masking neutralising epitopes by glycan shielding, and using decoy epitopes to prevent fast and effective humoral immune response (Lopez and Osorio, 2004; Mateu and Diaz, 2008).

The coding capacity of the PRRSV genome is maximised by an arrangement where the consecutive genes of the structural proteins are localised in alternating frames and they partially overlap with each other with the exception of ORF4 and ORF5 (Yu et al., 2009; Lunney et al., 2016). In addition, three accessory proteins were identified translating from fully overlapping ORFs by noncanonical translation mechanisms (Wu et al., 2001; Firth et al., 2011; Fang et al., 2012).

In this paper we report the identification by bioinformatic methods of a novel small protein (7ap) coding overlapping ORF (ORF7a) in the coding region of the $\mathrm{N}$ gene. Depending on the virus strain, the length of the protein is between 36 and 53 amino acids (aa). Translation of the ORF was proven by FLAG and GFP fusion constructs. 7ap binds DNA, RNA and the Fc part of the IgG, and it inhibits complement fixation in vitro. The potential functional implications of the findings are discussed.

\section{Materials and methods}

\section{Fusion constructs}

The pEGFP-N1 vector was used to fuse the three alternative frames of the nucleocapsid gene (ORF7) to GFP. In each construct approximately two thirds of the nucleocapsid gene of the PRRSV strain HU-14432/2011 was cloned, starting with the TRS sequence and ending with the last codon of the ORF7a. To amplify the cDNAs the $7 \mathrm{~F}$ common forward primer was used. The three reverse primers $7 \mathrm{r}, 7 \mathrm{ar}$ and $7 \mathrm{ncr}$ differed only in the addition of single nucleotides to shift the translational frames in the three fusion constructs. The PCR products were digested with XhoI and BamHI and were ligated into the same restriction sites of the vector. 
To create 3xFLAG constructs, first the pcDNA-FLAG vector was created by cloning the $3 x F L A G$ (DYKDHDGDYKDHDIDYKDDDDK) into pcDNA3 by digesting the vector with $\mathrm{XbaI}$ and $\mathrm{XhoI}$ and inserting the annealed 3 flagF and 3 flagR oligonucleotides.

To generate similar fusion constructs in pcDNA-FLAG as it was done in pEGFPN1, the 7Ff forward and the 7Rf, 7arf and the 7ncrf reverse primers were used.

The mutation in the start codon of eGFP (M1A) was executed on the pEGFP-N1 vector with the gfp_mut_fw and the gfp_mut_rev primers using the quick change site directed mutagenesis method (Papworth et al., 1996). To express 7ap as a fusion protein in the resulted p-MeGFPN1 plasmid ORF7a was amplified with F-gfp-orf7a and R-gfp-orf7a primers, digested with XhoI and BamHI enzymes and cloned into the same sites of the plasmid (Table 1).

All constructs were transfected into Marc 145 (Kim et al., 1993) and PT (Bergeron et al., 1993) cells with Turbofect (Thermo Scientific, Waltham, Massachusetts) according to the manufacturer's recommendation on 24-well cluster plates.

Table 1

List of primers (the nucleotides causing frameshift are underlined)

\begin{tabular}{ll}
\hline Primers & \\
\hline $7 \mathrm{~F}$ & GAGGATCCTTAACCTCGTCAAGTATGGCCG \\
$7 \mathrm{r}$ & GACTCGAGTGGCGCACTGTATGAGCAA \\
$7 \mathrm{ar}$ & GACTCGAGTTGGCGCACTGTATGAGCAA \\
$7 \mathrm{ncr}$ & GACTCGAGGGCGCACTGTATGAGCAA \\
3flagF & TCGAGTGACTACAAAGACCATGACGGTGATTATAAAGATCAT \\
& GACATCGACTACAAGGATGACGATGACAAGTAGTGAA \\
3flagR & CTAGTTCACTACTTGTCATCGTCATCCTTGTAGTCGAT \\
& GTCATGATCTTTATAATCACCGTCATGGTCTTGTAGCAC \\
7Ff & GACTCGAGTTAACCTCGTCAAGTATGGCCG \\
7Rf & GAGGATCCTGGCGCACTGTATGAGCAA \\
7arf & GAGGATCCTTGGCGCACTGTATGAGCAA \\
7ncrf & GAGGATCCGGCGCACTGTATGAGCAA \\
gfp_mut_fw & CCACCGGTCGCCACCGCGGTGAGCAAGGGCGAG \\
gfp_mut_rev & CTCGCCCTTGCTCACCGCGGTGGCGACCGGTGG \\
F-gfp-orf7a & CAACTCGAGATGACATCCGGCACCACCTC \\
\hline
\end{tabular}

Peptide synthesis

Hu7ap (MTSGTTSPRLNAPSACNRSRRLSIKAQELRRFHPAGRSVFR LSLCCRLLIQCA) and Wu7ap (MTSGITLPLVSGNCVCRRSRLPSIRVLELVP CQIQGG) were synthesised and purified to 95\% purity by CASLO ApS (Lyngby, Denmark). 


\section{Indirect immunofluorescence (IF)}

The cells were fixed with $300 \mu 13 \%$ formaldehyde solution and washed twice with $1 \mathrm{ml}$ washing buffer [phosphate-buffered saline (PBS) $0.1 \%$ Tween 20]. The samples were then incubated in $200 \mu 11 \%$ Triton-X (in PBS) for $15 \mathrm{~min}$ at room temperature. Next the samples were washed with washing buffer and incubated with $200 \mu \mathrm{l}$ primary antibodies (mouse anti-FLAG M2 monoclonal antibody 1000-fold dilution (Sigma-Aldrich, St. Louis, Missouri) and serially diluted mouse and pig sera in IF buffer (4\% horse serum in PBS). After incubation for $1 \mathrm{~h}$, the cells were washed twice with washing buffer, and $200-\mu 1$ volumes of secondary antibodies [CF594 goat anti-mouse and CF568 goat anti-swine IgGs (Biotium Hayward, California) in 1000-fold dilution] and Hoechst $33342(1 \mu \mathrm{g} / \mu \mathrm{l})$ were added. After washing, the samples were examined under a Zeiss Axio Observer D1 inverse fluorescence research microscope.

\section{Direct ELISA}

The plate was coated with $20 \mu \mathrm{g} / \mathrm{ml}$ ORF7a solution and incubated overnight at $4{ }^{\circ} \mathrm{C}$. Next day the plate was washed three times with washing buffer $(0.5 \%$ Tween 20 in PBS), and blocked for an hour with $200 \mu \mathrm{l}$ blocking buffer (1\% BSA/PBS solution) at $37^{\circ} \mathrm{C}$. The plates were then washed three times with washing buffer. The different HRP-conjugated antibodies [polyclonal swine antirabbit immunoglobulins/HRP (DAKO A/S, Glostrup, Denmark), goat anti-pig $\operatorname{IgG}(\mathrm{H} / \mathrm{L})(\mathrm{AbD}$ Serotec, Kidlington, UK), goat anti-chicken IgY $(\mathrm{H}+\mathrm{L})-\mathrm{HRP}$ (SouthernBiotech, Birmingham, Alabama), rabbit anti-pig IgG H\&L (HRP) (Abcam, Cambridge, UK), mouse anti-rabbit IgG-HRP (Santa Cruz Biotechnology, Dallas, Texas)] were serially diluted with blocking buffer and added to the wells. After 1-h incubation at $37^{\circ} \mathrm{C}$ the plates were washed four times and $100 \mu \mathrm{L}$ ABTS (2,2'-Azinobis [3-ethylbenzothiazoline-6-sulfonic acid]-diammonium salt) solution (Ingenasa, Madrid, Spain) was added. After 20-min incubation the reaction was stopped with $5 \%$ SDS. OD was measured at $410 \mathrm{~nm}$ with an ELISA reader (ELx800 ELISA plate reader, Dialab GmbH, Austria). The samples were investigated in triplicates and the averages of the OD values were calculated.

\section{Complement fixation inhibition test}

To set up the complement fixation system, $25 \mu 1$ of $2.5 \%$ freshly prepared sheep erythrocytes (Culex Bt, Budapest, Hungary) was mixed with $25 \mu 1$ 1:2000 diluted haemolytic amboceptor (rabbit anti-sheep erythrocytes IgG) (Virion/ Serion, Würzburg, Germany) and $25 \mu \mathrm{l}$ serially diluted (4-0.4\% in ten steps) guinea pig complement (Virion/Serion, Würzburg, Germany), and the samples were incubated for $30 \mathrm{~min}$ at $37^{\circ} \mathrm{C}$. All dilutions of the components and the reactions were executed in veronal buffer (Idexx Laboratories, Westbrook, Maine). The lowest complement dilution where full lysis occurred was chosen and, to ex- 
clude false positive results, one percent higher concentration complement was applied in the inhibition tests (so if full lysis was observed with $2 \%$ complement, then $3 \%$ was used in the inhibition test).

In the inhibition test staphylococcal protein $\mathrm{A}(\mathrm{spA})$ was used as negative control. All tested components including Hu7ap Wu7ap and spA were investigated in twofold serial dilution starting with $1.5 \mu \mathrm{g}, 6 \mu \mathrm{g}$ and $1.5 \mu \mathrm{g}$, respectively. For the test at first the haemolytic amboceptor was incubated for $1 \mathrm{~h}$ with the serially diluted peptides and spA at $37{ }^{\circ} \mathrm{C}$ in $25-\mu 1$ volumes, then the other components were added in similar concentrations and volumes as indicated above. Samples were incubated again for $30 \mathrm{~min}$ at $37^{\circ} \mathrm{C}$ and the results were analysed.

\section{Protein-protein gel retardation assay}

7ap was added to $8-10 \mu \mathrm{g}$ IgG or IgG fragments in $14 \mu \mathrm{l} 0.01 \mathrm{M}$ potassium phosphate, $75 \mathrm{mM}$ sodium chloride, $\mathrm{pH} 7.2$ and was incubated for $1 \mathrm{~h}$ at room temperature, then $6 \mu 1$ loading buffer was added and the samples were analysed by electrophoresis at $120 \mathrm{~V}$ for $2 \mathrm{~h}$ on a clear native polyacrylamide gel. The protein was stained with $0.1 \%$ Coomassie Brilliant Blue R250.

\section{Results}

\section{Identification of a small conserved ORF}

To identify unrecognised ORFs with potential translational products, bioinformatic examination of 46 sequentially divergent PRRSV genomes was performed.

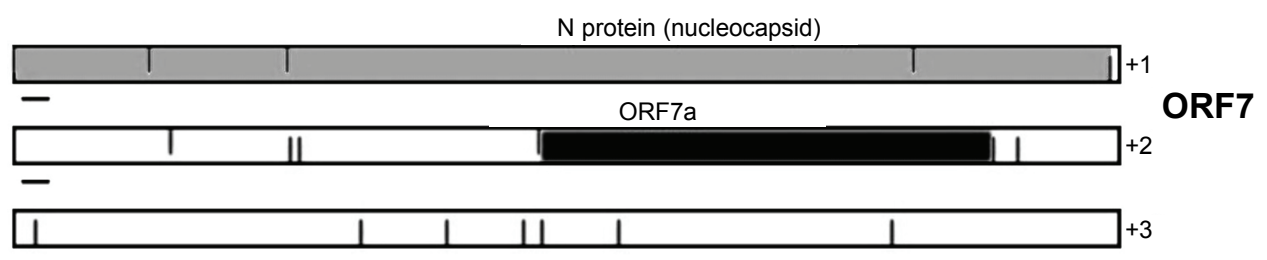

Fig. 1. Schematic representation of the three positive strand frames of the nucleocapsid coding region of PRRSV strain HU-14432/2011. The ORFs of interest are symbolised by shaded boxes.

+1 frame encodes the canonical protein (nucleocapsid), +2 frame encodes the ORF7a

Sequence analysis by the ORF finder tool (Tatusov) revealed a short alternative ORF named ORF7a (Fig. 1) within the nucleocapsid gene of all investigated virus genomes. ORF7a localised in the +2 frame containing a methionine codon in a conserved position and depending on genotypes continued in a coding region stretching between 26 and 53 aa. Alignment of the translated protein sequences (named 7ap) revealed five highly conserved and ten conserved aa in the 
protein (Fig. 2). 7aps from divergent strains have different physicochemical properties; however, they are all predicted to be positively charged at neutral $\mathrm{pH}$ as a result of componential imbalance between basic and acidic aa in favour of the former one.

HKEU16 (EU076704.1) KNU-07 (FJ349261.1) $\mathrm{SD} 01-08(\mathrm{DQ} 489311.1)$ BJEU06-1 (GU04734.1) BJE Amervac (GU067771. SHE ( GQ461593.1) Lelystad (M96262.2) HU-14432/2011 01CB1 (DQ864705.1) 07V063 (GU737264.2) IV3140 (DQ355822.1) Lena (JF802085.1) Soz-8 (DQ324720.1) Soz-8 (DQ324720.1) obu-1 (DQ324707.1) Yuz-48 (DQ324728.1) Zap-41 (DQ324734.1) Vos-29 (DQ324725.1) Pyrsvac-187 (DQ324712.1) Upa-13 (DQ324721.1) Dzi-64 (DQ324706.1) Che-46 (DQ324703.1) Che-4 (DQ324703.1) Vas-3 (DQ324723.1) WUH 4 ( VR-2332 (AY150564.1) MN30100 (EF536000.1) NVDC-JS2-2011 (JQ715698.1) NVSL 97-7985 (AF325691.1) $\mathrm{CH}-1 \mathrm{a}$ (AY032626.1) Hawkeye 7 (EF532815.1) Flagship (EF532805.1) JA-142 (AF396844.1) Yn-2011 (JX857698.1) A2MC2 (JQ087873.1) MN184B (DQ176020.1) SDSU73 (JN654458.1) P129 (AF494042.1) 17198-6 (EF442776.1) CA (FJ194950.1) $05 K 212$ (EF441810.1) PL97-1/LP1 (AY612613.1) JXwn06-81C (HO233604.1) SX-1 (GQ857656.1) NMEU09-1(GU047345.1) Aus (AF438362.1)

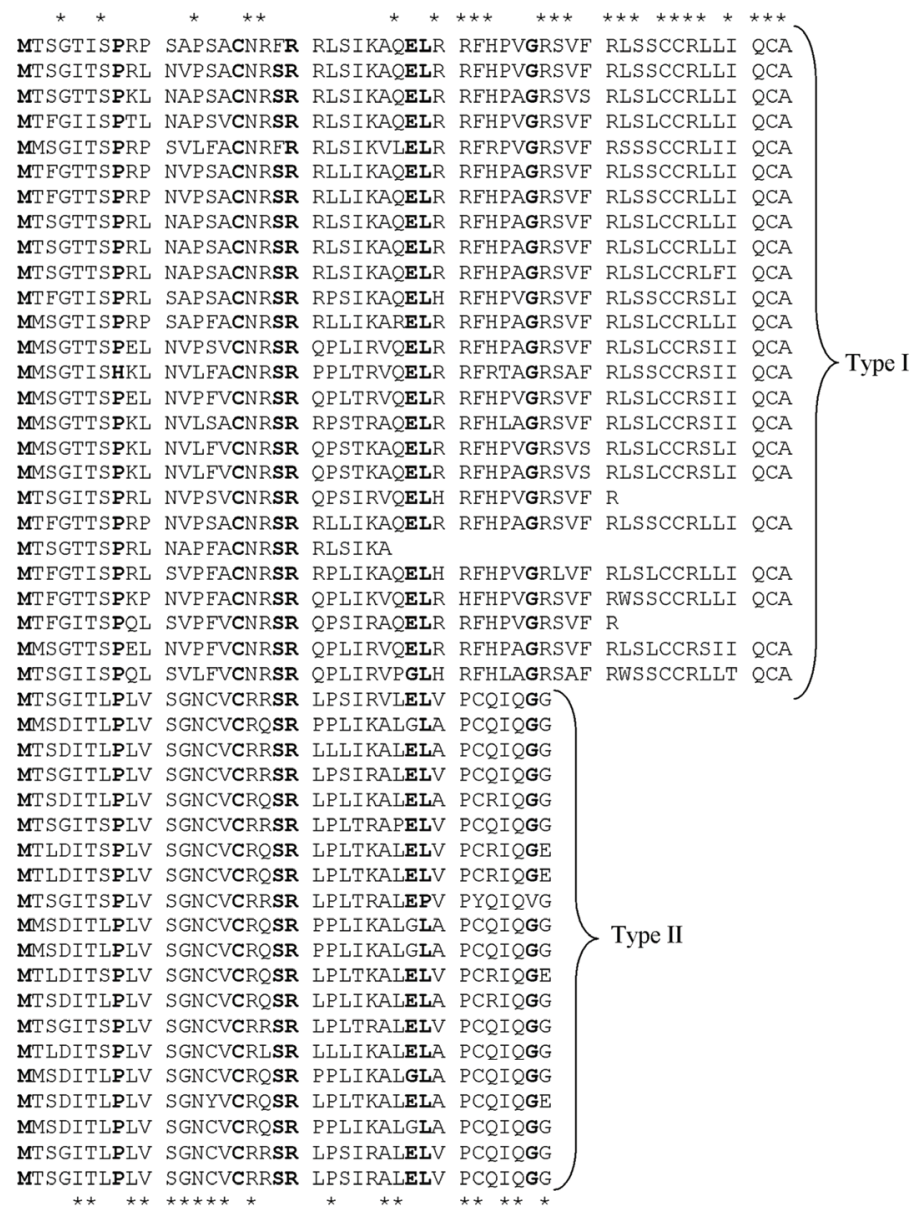

Fig. 2. Alignment of 7ap sequences. Accession numbers of the different strains are in parentheses. Amino acids in bold are conserved in both genotypes, the asterisks in the first and last rows label conserved amino acids in type I and type II sequences, respectively

\section{Translation and localisation of 7 ap}

The most plausible explanation of the universal presence of ORF7a and the observed conservations of the coded proteins in sequence and physicochemical characteristics is that ORF7a encodes a protein. To demonstrate the existence of 7 ap and to investigate the translation mechanism of ORF7a eGFP fusion constructs were created by cloning the cDNA of the 5' half the ORF7 mRNA (starting 
with the translational regulator sequence and finishing with the last codon of 7ap) of the HU-14432/2011 PRRSV strain into the pEGFPN1 vector in the three consecutive frames (Fig. 3A-B). The constructs were transfected into Marc-145 cells and, after $24 \mathrm{~h}$, the cells were fixed and monitored for eGFP expression. As expected, the positive control construct, in which the eGFP was inserted into frame +1 coding the nucleocapsid protein, resulted in a robust fluorescent signal in the cells. Also, strong fluorescent signal was detected, although with lower intensity, in the PT cells when eGFP was fused to the last codon of 7ap in frame +2 indicating the translation of ORF7a. Interestingly, low levels of eGFP signal could also be detected at high-intensity excitation in cells transfected by the fusion constructs containing the eGFP in frame +3 (Fig. 3C).

To confirm the translation of 7ap and to exclude that the detected fluorescent signal in the frame +2 construct was the result of artificial initiation of the eGFP translation from its own ATG codon, other plasmid constructs were generated, where the eGFP fusion tag in the three different frames was replaced by a triple FLAG tag. These constructs were also transfected into PT cells, which were monitored for FLAG expression $28 \mathrm{~h}$ post transfection. FLAG expression could be detected in cells transfected by the frame +1 and frame +2 constructs, verifying that 7ap is in fact translated from the nucleocapsid mRNA (Fig. 3D).

To facilitate background-free tracing of 7ap in the cell, first the pEGFPN1 vector (p-MeGFPN1) was modified by mutating the start codon of eGFP from ATG (M) to GCG (A). This mutation prevented the translation of eGFP from its own start codon (M1AeGFP), but allowed initiation from a fusion partner and the translation of M1AeGFP as a fluorescently active fusion protein. Second, ORF7a was cloned into p-MeGFPN1 by fusing 7ap to M1AeGFP (7apM1AeGFP). This plasmid construct was transfected into Marc 145 cells and the 7ap-M1AeGFP signal was followed in the transfected cells. Soon (16-20 h) after transfection, the majority of the fluorescent signal appeared in the nucleus, while later it could be detected in the whole cell, indicating that 7ap is first transported into the nucleus and later it also accumulates in the cytoplasm (Fig. 3E).

\section{IgG binding and inhibition of complement fixation}

For serological detection of 7ap ORF7a of the HU-14432/2011 strain was expressed in E. coli by two different expression systems, pet28b (Merck KgaA, Darmstadt, Germany) and pBAD2TEV (Zádori et al., 2001). In both systems the expressed protein proved to be highly toxic to the bacteria and purification of bacterially expressed 7ap remained unsuccessful despite several attempts (data not shown).

To complete the serological investigation of PRRSV-positive animals, 7ap of HU-14432/2011 (Hu7ap) was synthesised chemically and used for coating the plate in a standard indirect ELISA test. PRRSV-positive sera (they were found positive in 1000 - to 2000 -fold dilution by INGEZIM PRRS Universal kit) were 

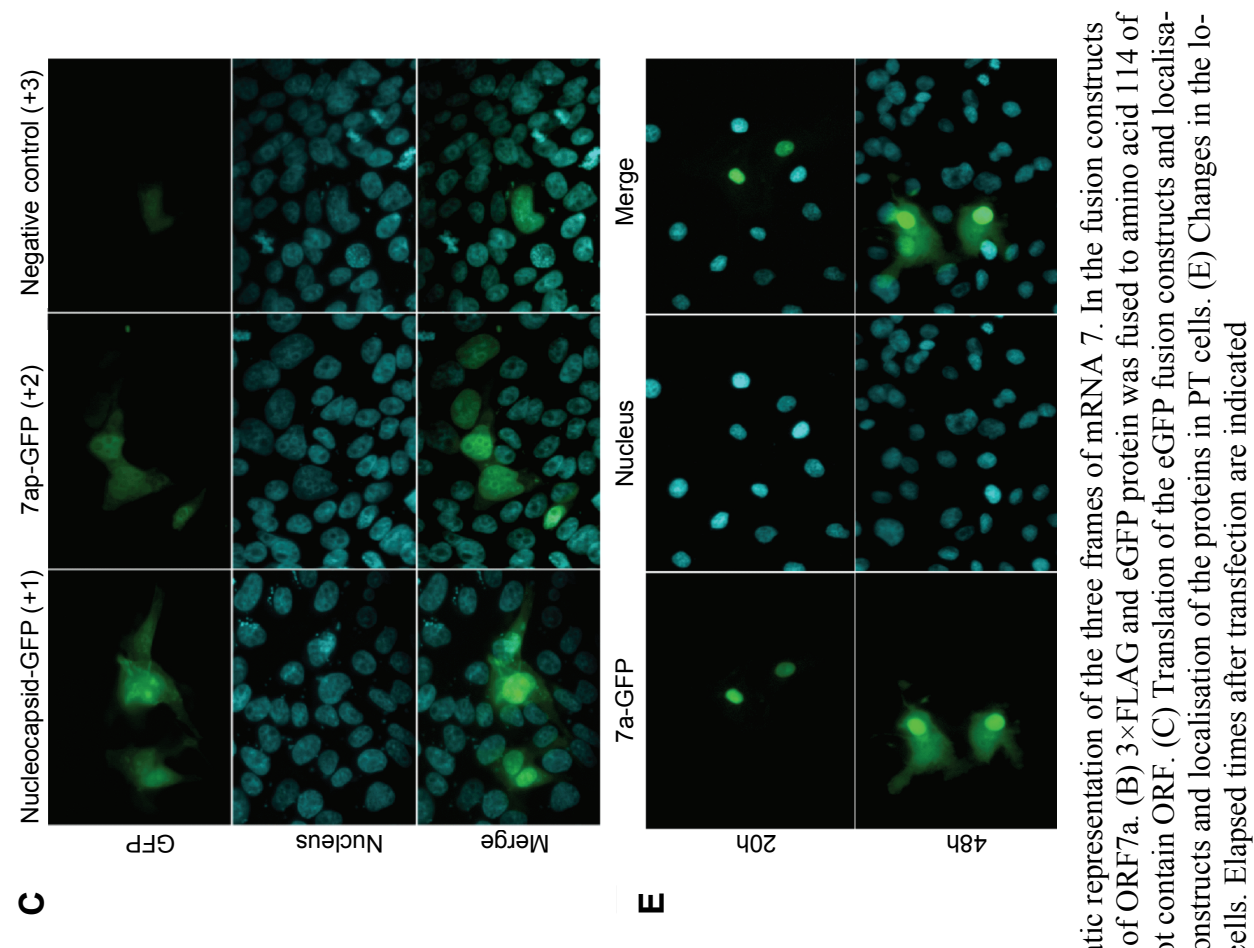

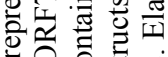
.

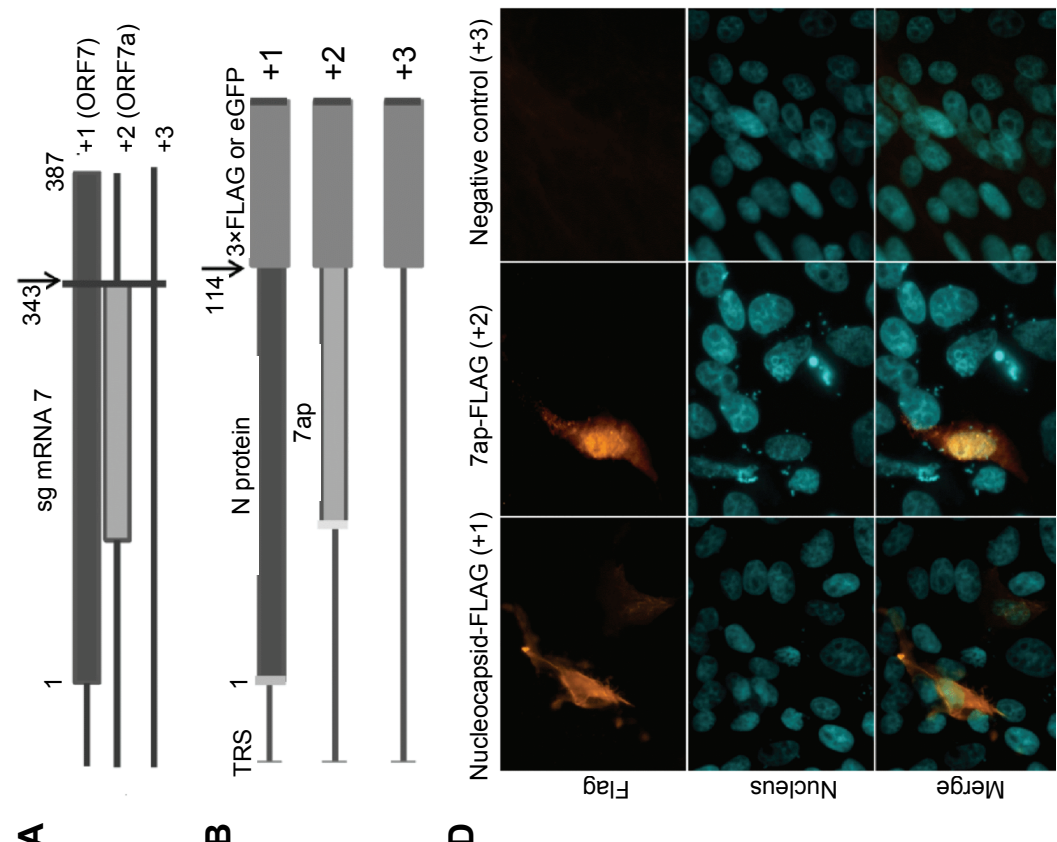

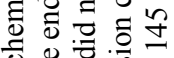
o 0

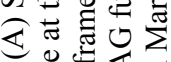

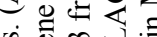

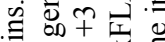
乙这离手

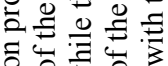
응 30 先学.몽 बित्ञ

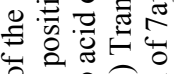

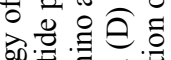

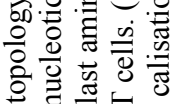
票焉

ते टु

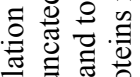

预芯离 的令范

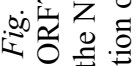


produced by intranasal infection of four pregnant sows with the HU-14432/2011 (GenBank accession number: KR296711.1) pathogenic strain. In the experiment, the sera of a non-infected pig and Hu7ap-coated wells without primary antibody treatment were also probed as standard negative controls.

Surprisingly, strong HRP signals were detected in all Hu7ap-coated wells, regardless of the presence or absence of primary sera (including the one from a non-infected animal). This finding suggested an 'aspecific' antigen-binding region $[\mathrm{F}(\mathrm{ab})]$, independent of the IgG binding capability of $7 \mathrm{ap}$.

To explore the nature of binding, several other mammalian IgG HRP conjugates were also investigated by ELISA. These experiments revealed that Hu7ap binds strongly to all investigated mammalian (pig, mouse, goat, and rabbit) IgG horseradish peroxidase (HRP) conjugates and that the binding is independent of HRP (Table 2).

Table 2

IgG binding capability of 7ap measured by direct ELISA

\begin{tabular}{lccccc}
\hline & \multicolumn{5}{c}{ Dilutions of sera } \\
\cline { 2 - 5 } IgG-HRP conjugates & $1000 \times$ & $2000 \times$ & $4000 \times$ & $8000 \times$ & $\begin{array}{c}1000 \times \\
\text { not 7ap coated }\end{array}$ \\
\hline Goat anti-chicken IgG & ++ & + & $+/-$ & - & - \\
Goat anti-pig IgG & ++ & ++ & + & + & - \\
Swine anti-rabbit IgG & + & + & $+/-$ & - & - \\
Rabbit anti-pig IgG & ++ & + & +- & - & - \\
Mouse anti-rabbit IgG & ++ & - & - & - & - \\
HRP & - & - & & - & - \\
\hline
\end{tabular}

$+++: \mathrm{OD}>1.6,++: \mathrm{OD}=1.59-0.8,+: \mathrm{OD}=0.79-0.4,+/-: \mathrm{OD}=0.39-0.2,-: \mathrm{OD}<0.19$

Protein-protein gel retardation assay (Park and Raines, 2004) was performed with Hu7ap to confirm the results of ELISA and to localise the binding site/s of 7ap on swine IgG. To explore whether a sequentially divergent 7ap possesses similar binding characteristics as Hu7ap, the 7ap of a type II PRRSV strain, WuH4 (Wu7ap) was also synthesised and analysed. Both Hu7ap and Wu7ap are positively charged peptides (pI 11.7 and 9.3, respectively) at near neutral $\mathrm{pH}$, so under native electrophoretic conditions they move in the opposite direction (toward the cathode) than the negatively charged IgGs and their fragments. The interaction between the negatively charged proteins and the positively charged peptides may result in complete inhibition of gel entry of the negatively charged protein as a consequence of increased size and decreased net negative charge of the complex.

In fact, $4 \mu \mathrm{g}$ and $20 \mu \mathrm{g}$ of $\mathrm{Hu} 7 \mathrm{ap}$ and $\mathrm{Wu} 7 \mathrm{ap}$ were able to completely inhibit the entry of $10 \mu \mathrm{g}$ of the monoclonal mouse IgG into the gel and twice as much was needed ( $8 \mu \mathrm{g}$ and $40 \mu \mathrm{g}$, respectively) to titrate out $10 \mu \mathrm{g}$ polyclonal pig IgG. 


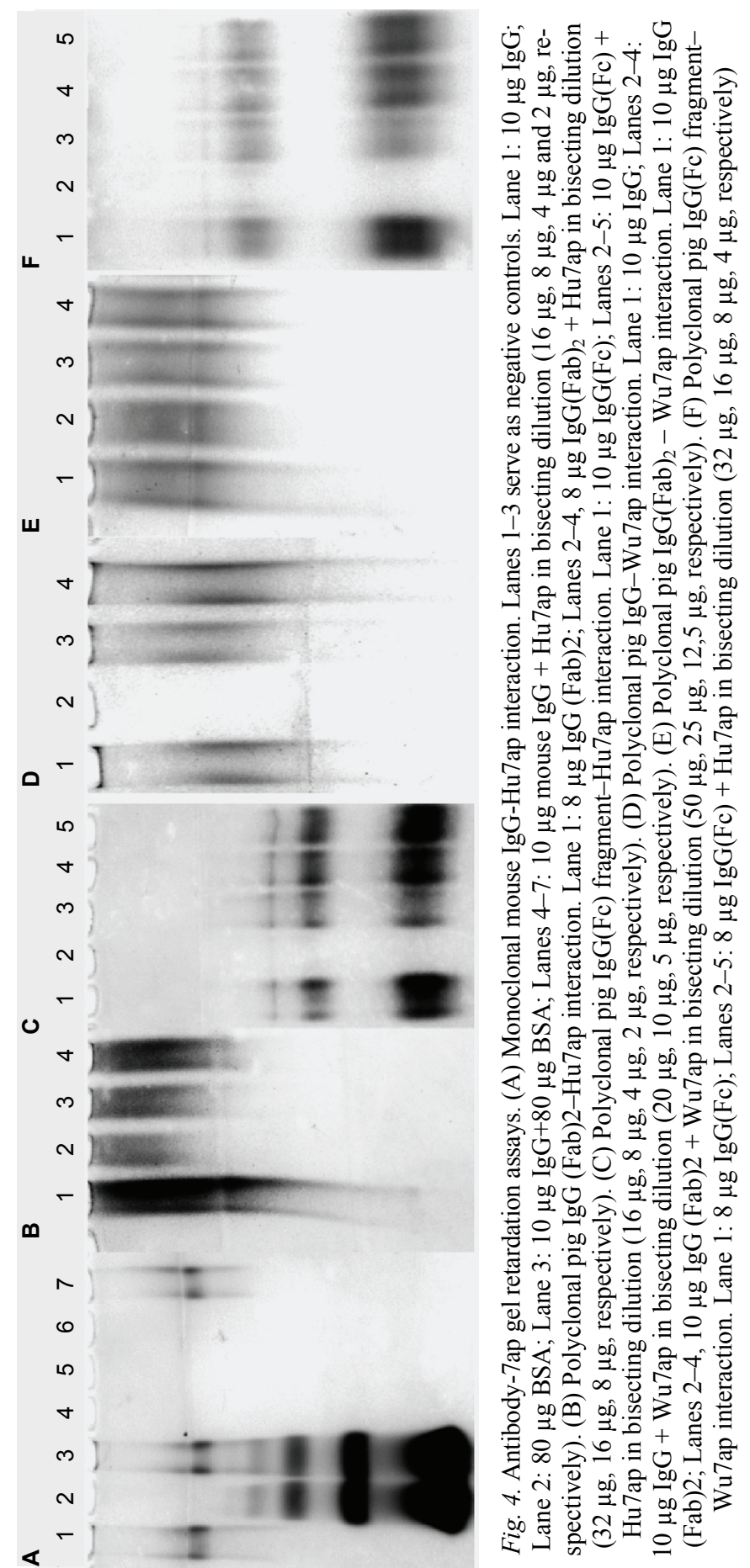


Similarly, applying in increasing concentrations $16 \mu \mathrm{g}$ and $32 \mu \mathrm{g}$ of Hu7ap and Wu7ap titrated out completely $10 \mu \mathrm{g}$ polyclonal pig $\operatorname{IgG}(\mathrm{Fc})$ fragment, while only a small fraction of the pig polyclonal $\operatorname{IgG}(\mathrm{Fab})_{2}$ was blocked running into the gel even when Hu7ap and Wu7ap were applied in large excess (Fig. 4). These experiments verified that despite their sequence heterogeneity, both 7aps are able to bind both pig and mouse IgGs and the main binding site of swine IgG is localised on the $\mathrm{Fc}$ fragment.

To further characterise the 7ap binding site, additional experiments were performed. SpA binds to the $\mathrm{CH} 3$ domain of the $\mathrm{Fc}$ fragment while $\mathrm{C} 1 \mathrm{q}$ of the complement system binds to the $\mathrm{CH} 2$ domain. In a competitive ELISA test 7aps applied in high concentrations did not inhibit spA-HRP binding to polyclonal pig $\operatorname{Ig}(\mathrm{Fc})$ (data not shown). However, in a standard complement fixation experiment, when anti-sRBC rabbit IgG (haemolysin) was preincubated with Hu7ap and Wu7ap, concentration-dependent inhibition of the haemolysis by the former one was observed, indicating the interference of $\mathrm{Hu} 7 \mathrm{ap}$ with $\mathrm{Clq}$ binding to $\mathrm{Fc}$ (Table 3). These experiments suggest that $\mathrm{Hu} 7$ ap binds to the $\mathrm{CH} 2$ domain of the $\mathrm{Fc}$, and its binding site at least partially overlaps with the C1q binding site.

Table 3

Complement fixation inhibition of different proteins

\begin{tabular}{lccccc}
\hline Proteins & $\begin{array}{c}\text { Concentration, } \\
\text { ng/ } \mu \mathrm{l}\end{array}$ & \multicolumn{5}{c}{ Dilutions of proteins } \\
\cline { 3 - 6 } & 15 & + & $2 \times$ & $4 \times$ & $8 \times$ \\
\hline Hu7ap & 15 & + & + & - \\
Wu7ap & 60 & - & - & - & - \\
spA & 15 & - & - & - & - \\
\hline
\end{tabular}

+: inhibition, - : no inhibition

\section{Discussion}

The nucleotide identity between the two PRRSV serotypes is only 55$70 \%$ and, according to one calculation, the mutation rate of PRRSV is 4.7-9.8 $\times$ $10^{-2} /$ nucleotide/year (Jenkins et al., 2002; Hanada et al., 2005), which would make it the highest reported among RNA viruses. However, more conservative estimations put the mutation rate somewhere between 1.8 and $7 \times 10^{-3} /$ nucleotide/year (Chang et al., 2002; Forsberg, 2005), which still places PRRSV among the most rapidly evolving viruses known. Despite the rapidly changing codon sequences of the main ORFs, recently several positionally conserved alternative ORFs have been identified in the PRRSV genome and all of them proved to be protein coding: ORFs partially coding the nsp2TF and nsp2N proteins in the NSP2 region (Fang et al., 2012), ORF5a and ORF2b overlapping with the ORF5 and ORF2a 
genes (Wu et al., 2001; Firth et al., 2011), respectively. Genome comparison of 46 divergent PRRSV genomes revealed another short ORF in a conserved position, named ORF7a overlapping with the ORF7 gene. Study of GFP and FLAG fusion constructs of the cDNA of the ORF7 region of the HU-14432/2011 PRRSV strain verified the translation of ORF7a. The conserved position of a methionine at the beginning of ORF7a in different PRRSV strains suggests start codon initiation of the ORF7ap in all PRRSV strains.

The translation of alternative frames in the nucleocapsid gene of several other nidoviruses [SARSV (Meier et al., 2006), bovine coronavirus 1 (Senanayake and Brian, 1997) and mouse hepatitis virus (MHV) (Fischer et al., 1997)] belonging to the genus Betacoronavirus was reported previously. The so-called I protein of MHV is a structural protein and its absence leads to reduced viral growth in tissue culture (Fischer et al., 1997), while ORF-9B of SARSV localises to mitochondria, binds to PCBP2 and AIP4, induces autophagy and severely limits host cell IFN responses (Shi et al., 2014). Overprinting of the nucleocapsid gene with an alternative ORF is not restricted to nidoviruses, since it can also be found at the core protein of the hepatitis $\mathrm{C}$ virus [Alternate Reading Frame Protein (ARFP)]. HCV ARFP stimulates apoptosis in plasmacytoid dendritic cells (Samrat et al., 2014), and it induces a cytokine imbalance in favour of immunosuppression by inhibiting IFN- $\alpha$ secretion and the production of IL-10 (Xu et al., 2014). Although the function of the alternative frame proteins is different even in related viruses, they all appear to promote viral spreading either directly as viral proteins or indirectly as immunosuppressive accessory proteins.

The remarkable biochemical characteristics of PRRSV 7ap suggest an immunosuppressive function as well. 7ap binds to the Fc part of mammalian IgGs and inhibits complement activation. In the classical pathway of complement activation the triggering event is the binding of the globular head of the $\mathrm{Clq}$ to the $\mathrm{CH} 2$ domain of IgG. On the $\mathrm{C} 1 \mathrm{q}$ heterotrimer several basic residues ( 7 arginines and one lysine and histidine each) have been identified to participate in residue-residue interaction with the Fc domain of IgG (Schneider and Zacharias, 2012). In addition, $\mathrm{Arg}^{\mathrm{B} 114}$ and $\mathrm{Arg}^{\mathrm{B} 129}$ of the $\mathrm{Clq}$ complex were proposed to mediate IgG recognition (Marqués et al., 1993). Considering its strong positive charge and the high number of arginine residues in 7ap, it is tempting to speculate that 7ap binds to aa of the $\mathrm{CH} 2$ domain, contributing to the $\mathrm{Fc} \mathrm{C} 1 \mathrm{q}$ interface, and this binding inhibits $\mathrm{Fc} \mathrm{Clq}$ interaction and complement fixation.

Several viral proteins have been identified in members of different virus families (Poxviridae, Retroviridae, Herpesviridae) that inhibit virus neutralisation by interaction with the components of the complement pathway (Hulo et al., 2011a). Binding of Fc receptor-like viral proteins to the $\mathrm{Fc}$ domain of $\mathrm{IgG}$ with immunosuppressive effects was also reported from several viral species (HCV, HHV-5, MCMV) (Hulo et al., 2011b). However, to our knowledge PRRSV 7ap is the first reported viral protein which binds to the Fc domain of IgG and inhib- 
its complement activation. Presently it is not clear when and where 7ap interacts with IgG during viral infection. We were unable to detect in vitro secretion of the 7ap-eGFP fusion protein from the supernatant of transfected cells by fluorimetric measurements (data not shown). However, externalisation by cell lysis or in vivo secretion of 7ap from infected macrophages cannot be excluded and it may ensure interaction.

Direct interactions of 7ap with intracellular proteins cannot be excluded either, particularly because most of the cytosolic proteins have net negative charges (Schwartz et al., 2001), while several regulating proteins interacting with them, like 7ap, are significantly shorter and have strong basic characters (Kiraga et al., 2007). It was shown that HIV-1 Tat, a similarly short viral basic protein, is able to bind to several regulator proteins and its binding to the cytosolic IkB-a depends on the arginine-rich region of the protein (residues 48-60) (Vitagliano et al., 2011).

During its evolution, PRRSV developed several molecular tools to counteract host responses and to evade the host immune system. Localisation and the remarkable immunological features of 7ap indicate that this protein is also one of such viral tools. Although our research revealed a substantial amount of data about the biochemical characteristics of $7 \mathrm{ap}$, further in vitro and in vivo studies are needed to clarify its translation in macrophages and its functional role in the life cycle of the virus.

\section{Acknowledgements}

This study was supported by the 'János Bolyai' Research Scholarship and by the Hungarian Scientific Research Fund (OTKA project K-108607).

\section{References}

Bergeron, J., Menezes, J. and Tijssen, P. (1993): Genomic organization and mapping of transcription and translation products of the NADL-2 strain of porcine parvovirus. Virology 197, 86-98.

Chang, C. C., Yoon, K. J., Zimmerman, J. J., Harmon, K. M., Dixon, P. M., Dvorak, C. M. and Murtaugh, M. P. (2002): Evolution of porcine reproductive and respiratory syndrome virus during sequential passages in pigs. J. Virol. 76, 4750-4763.

Charerntantanakul, W. (2012): Porcine reproductive and respiratory syndrome virus vaccines: Immunogenicity, efficacy and safety aspects. World J. Virol. 12, 23-30.

Fang, Y., Treffers, E. E., Li, Y., Tas, A., Sun, Z., van der Meer, Y., de Ru, A. H., van Veelen, P. A., Atkins, J. F., Snijder, E. J. and Firth, A. E. (2012): Efficient -2 frameshifting by mammalian ribosomes to synthesize an additional arterivirus protein. Proc. Natl Acad. Sci. U. S. A. 109, E2920-E2928. doi: 10.1073/pnas.1211145109.

Firth, A. E., Zevenhoven-Dobbe, J. C., Wills, N. M., Go, Y. Y., Balasuriya, U. B. R., Atkins, J. F., Snijder, E. J. and Posthuma, C. C. (2011): Discovery of a small arterivirus gene that over- 
laps the GP5 coding sequence and is important for virus production. J. Gen. Virol. 92, 1097-1106.

Fischer, F., Peng, D., Hingley, S. T., Weiss, S. R. and Masters, P. S. (1997): The internal open reading frame within the nucleocapsid gene of mouse hepatitis virus encodes a structural protein that is not essential for viral replication. J. Virol. 71, 996-1003.

Forsberg, R. (2005): Divergence time of porcine reproductive and respiratory syndrome virus subtypes. Mol. Biol. Evol. 22, 2131-2134.

Hanada, K., Suzuki, Y., Nakane, T., Hirose, O. and Gojobori, T. (2005): The origin and evolution of porcine reproductive and respiratory syndrome viruses. Mol. Biol. Evol. 22, 1024-1031.

Hulo, C., de Castro, E., Masson, P., Bougueleret, L., Bairoch, A., Xenarios, I. and Le Mercier, P. (2011a): ViralZone: a knowledge resource to understand virus diversity. Nucleic Acids Res 39, D576-582. doi: 10.1093/nar/gkq901. http://viralzone.expasy.org/all_by_protein/811.html (April 4, 2015).

Hulo, C., de Castro, E., Masson, P., Bougueleret, L., Bairoch, A., Xenarios, I. and Le Mercier, P. (2011b): ViralZone: a knowledge resource to understand virus diversity. Nucleic Acids Res 39:D576-582. doi: 10.1093/nar/gkq901. http://viralzone.expasy.org/all_by_species/812.html\#tab6 (April 4, 2015).

Jenkins, G. M., Rambaut, A., Pybus, O. G. and Holmes, E. C. (2002): Rates of molecular evolution in RNA viruses: a quantitative phylogenetic analysis. J. Mol. Evol. 54, 156-165.

Kim, H. S., Kwang, J., Yoon, I. J., Joo, H. S. and Frey, M. L. (1993): Enhanced replication of porcine reproductive and respiratory syndrome (PRRS) virus in a homogeneous subpopulation of MA-104 cell line. Arch. Virol. 133, 477-483.

Kiraga, J., Mackiewicz, P., Mackiewicz, D., Kowalczuk, M., Biecek, P., Polak, N., Smolarczyk, K., Dudek, M. R. and Cebrat, S. (2007): The relationships between the isoelectric point and: length of proteins, taxonomy and ecology of organisms. BMC Genomics 8, 163.

Lopez, O. J. and Osorio, F. A. (2004): Role of neutralizing antibodies in PRRSV protective immunity. Vet. Immunol. Immunopathol. 102, 155-163.

Lunney, J. K., Fang, Y., Ladinig, A., Chen, N., Li, Y., Rowland, B. and Renukaradhya, G. J. (2016): Porcine reproductive and respiratory syndrome virus (PRRSV): Pathogenesis and interaction with the immune system Annu. Rev. Anim. Biosci. 4, 129-154.

Marqués, G., Antón, L. C., Barrio, E., Sánchez, A., Ruiz, S., Gavilanes, F. and Vivanco, F. (1993): Arginine residues of the globular regions of human $\mathrm{Clq}$ involved in the interaction with immunoglobulin G. J. Biol. Chem. 268, 10393-10402.

Mateu, E. and Diaz, I. (2008): The challenge of PRRS immunology. Vet. J. 177, 345-351.

Meier, C., Aricescu, A. R., Assenberg, R., Aplin, R. T., Gilbert, R. J., Grimes, J. M. and Stuart, D. I. (2006): The crystal structure of ORF-9b, a lipid binding protein from the SARS coronavirus. Structure 14, 1157-1165.

Murtaugh, M. P., Stadejek, T., Abrahante, J. E., Lam, T. T. and Leung, F. C. (2010): The everexpanding diversity of porcine reproductive and respiratory syndrome virus. Virus Res. 154, 18-30.

Papworth, C., Bauer, J. C., Braman, J. and Wright, D. A. (1996): Site-directed mutagenesis in one day with $>80 \%$ efficiency. Strategies 9, 3-4.

Park, S. H. and Raines, R. T. (2004): Fluorescence gel retardation assay to detect protein-protein interactions. Methods Mol. Biol. 261, 155-160.

Samrat, S. K., Li, W., Singh, S., Kumar, R. and Agrawal, B. (2014): Alternate reading frame protein (F protein) of hepatitis $\mathrm{C}$ virus: paradoxical effects of activation and apoptosis on human dendritic cells lead to stimulation of T cells. PLoS One 9, e86567. doi: 10.1371/ journal.pone.0086567.

Schneider, S. and Zacharias, M. (2012): Atomic resolution model of the antibody Fc interaction with the complement C1q component. Mol. Immunol. 51, 66-72. 
Schwartz, R., Ting, C. S. and King, J. (2001): Whole proteome pI values correlate with subcellular localizations of proteins for organisms within the three domains of life. Genome Res. 11, 703-709.

Senanayake, S. D. and Brian, D. A. (1997): Bovine coronavirus I protein synthesis follows ribosomal scanning on the bicistronic N mRNA. Virus Res. 48, 101-105.

Shi, M., Lam, T. T. Y., Hon, C. C., Murtaugh, M. P., Davies, P. R., Hui, R. K. H., Li, J., Wong, L. T. W., Yip, C. W., Jiang, J. W. and Leung, F. C. C. (2010): Phylogeny-based evolutionary, demographical, and geographical dissection of North American type 2 porcine reproductive and respiratory syndrome viruses. J. Virol. 84, 8700-8711.

Stadejek, T., Oleksiewicz, M. B., Potapchuk, D. and Podgórska, K. (2006): Porcine reproductive and respiratory syndrome virus strains of exceptional diversity in eastern Europe support the definition of new genetic subtypes. J. Gen. Virol. 87, 1835-1841.

Tatusov, T. and Tatusov, R. NCBI ORF Finder. http://www.ncbi.nlm.nih.gov/projects/gorf/.

Tóth, R., Mészáros, I., Farsang, A. and Zádori, Z. (2016): Mechanisms of action of veterinary vaccine adjuvants (in Hungarian, with English abstract). Magy. Allatorvosok 138, 31-46.

Vitagliano, L., Fiume, G., Scognamiglio, P. L., Doti, N., Cannavò, R., Puca, A., Pedone, C., Scala, G., Quinto, I. and Marasco, D. (2011): Structural and functional insights into IאB- $\alpha / \mathrm{HIV}-1$ Tat interaction. Biochimie 93, 1592-1600.

Wu, W. H., Fang, Y., Farwell, R., Steffen-Bien, M., Rowland, R. R. R., Christopher-Hennings, J. and Nelson, E. A. (2001): 10-kDa structural protein of porcine reproductive and respiratory syndrome virus encoded by ORF2b. Virol. 287, 183-189.

Xu, X., Yu, X., Deng, X., Yue, M., Zhang, J., Zhu, D., Zhou, Z., Zhai, X., Xu, K. and Zhang, Y. (2014): Hepatitis $C$ virus alternate reading frame protein decreases interferon- $\alpha$ secretion in peripheral blood mononuclear cells. Mol. Med. Rep. 9, 730-736.

Yu, D., Lv, J., Sun, Z., Zheng, H., Lu, J. and Yuan, S. (2009): Reverse genetic manipulation of the overlapping coding regions for structural proteins of the type II porcine reproductive and respiratory syndrome virus. Virol. 383, 22-31.

Zádori, Z., Szelei, J., Lacoste, M. C., Li, Y., Gariépy, S., Raymond, P., Allaire, M., Nabi, I. R. and Tijssen, P. (2001): A viral phospholipase A2 is required for parvovirus infectivity. Dev. Cell 1, 291-302.

Zuckermann, F. A., Garcia, E. A., Luque, I. D., Christopher-Hennings, J., Doster, A., Brito, M. and Osorio, F. (2007): Assessment of the efficacy of commercial porcine reproductive and respiratory syndrome virus (PRRSV) vaccines based on measurement of serologic response, frequency of gamma-IFN-producing cells and virological parameters of protection upon challenge. Vet. Microbiol. 123, 69-85. 